\title{
Sleep quality of higher education students during COVID-19 and its association with diet quality and lifestyle behaviours
}

\author{
S. Doak ${ }^{1}$, J. Kearney ${ }^{2}$, J.M. McCormack ${ }^{1}$ and L. Keaver ${ }^{1}$ \\ ${ }^{1}$ Department of Health and Nutritional Science, Institute of Technology Sligo, Sligo, Ireland and \\ ${ }^{2}$ School of Biological and Health Sciences, Technological University Dublin, City Campus, Dublin 8, Ireland
}

Many lifelong health-related behaviours are established transitioning from school into higher education including a decline in sleep ${ }^{(1)}$ and diet quality ${ }^{(2)}$; likely to be maintained throughout life, affecting future health status ${ }^{(3)}$. Inadequate sleep quality and quantity can contribute to public health concerns as it is associated with poor dietary behaviours ${ }^{(2)}$ and unhealthy lifestyle behaviours ${ }^{(3,4)}$.

Recognising sleep, dietary and lifestyle behavioural patterns in students may provide evidence for healthy campus initiatives. The aim of this study was to assess the current sleep quality and its relationship with diet and lifestyle behaviours in a sample of Irish higher education students.

Recruitment was during the 2020-2021 academic year with full-time students, aged 18+, eligible to participate in this online crosssectional study, hosted by Qualtrics ${ } X \mathrm{XM}$. Self-reported questions were asked in relation to demographics, BMI, smoking, and the impact of COVID-19. Sleep quality of students was measured using the validated Pittsburgh Sleep Quality Index - (PSQI), and four validated measures to determine dietary quality (short-form food frequency questionnaire), alcohol use (Alcohol Use Disorders Identification Test Alcohol Consumption - AUDIT-C), perceived stress (Perceived Stress Scale-10 - PSS-10), and physical activity levels (International Physical Activity Questionnaire Short Form - IPAQ-SF). Statistical analysis was performed using Pearson's product- moment correlation and a one-way ANOVA test. Significance was set at $\mathrm{p}<0.05$.

The cohort $(n=682)$ had a mean age of $24.97 \pm 8.90$ and $66.8 \%$ were female. Mean PSQI scores and hours slept per night were $4.70 \pm 2.46$ and $7.16 \pm 1.25$, respectively, indicating that $45.2 \%(n=304)$ have poor sleep quality and $33.0 \%(n=223)$ do not meet the recommended sleep amount of $7+$ hours.

COVID-19 negatively impacted the sleep of 39.0\% $(n=266)$ of students. There was a statistically significant, small correlation found between PSQI and PSS-10 (stress) scores $(r(672)=0.195, p<0.001)$, AUDIT-C scores $(r(672)=0.118, p=0.002)$ and a negative correlation with IPAQ scores $(r(655)=-0.154, p<0.001)$ and diet quality scores $(r(672)=-0.115, p=0.003)$. A statistically significant increase was found in the mean PSQI of smokers compared to non-smokers $(F(3,667)=8.158,1.45,95 \% C I(0.66$ to 2.25), $p<0.001)$, but not ex- or social- smokers. There was no statistically significant correlation between PSQI scores and fruit and vegetable $(\mathrm{F} \& \mathrm{~V})$ consumption $(p=0.176)$ or BMI $(p=0.344)$.

There is evidence of a correlation between having better sleep quality and a higher quality diet, being more physically active, consuming less alcohol, having lower stress levels, and not being a smoker. BMI and F\&V consumption were not associated with sleep quality. Sleep quality is an important determinant arising from recent international research ${ }^{(4)}$ and further research to assess sleeps correlation with diet and lifestyle behaviours are warranted in this population Group ${ }^{(3)}$. These findings highlight the importance of the inclusion of sleep within health promotion campaigns among students.

\section{Acknowledgments}

The authors wish to thank Jason Quinn, Keelan Kennoy and Ryan O'Reilly from ITS Students' Union, Yvonne Roache from ITS, Colin Kearney and Victor O'Loughlin from GMIT Students' Union and Mary Hernandez from LYIT Students' Union for helping with survey promotion. In addition, the authors thank the students who participated in this study as volunteers, making the study possible.

\section{References}

1. Park H, Chiang JJ, Irwin MR et al. (2019) Sleep Med 60, 202-210.

2. Aslan Çin NN \& Yardimci H (2021) Sleep Biol Rhythms 19, 313-323.

3. Gherasim A, Arhire LI, Nită O, et al. (2020) Proc Nutr Soc 79(3), 311-323.

4. Wang F \& Bíró É (2021) Éxplore 17(2),170-177. 